\title{
The long and winding road: from genetic risk factors to the understanding of disease-pathogenesis in Crohn's disease
}

\author{
Gerhard Rogler ${ }^{1} \cdot$ Martin Hausmann ${ }^{1}$
}

Received: 23 January 2019 / Accepted: 23 January 2019 / Published online: 18 February 2019

(c) Springer Nature Limited 2019

Crohn's disease $(\mathrm{CD})$ is a chronic, relapsing inflammatory disorder of the intestinal mucosa usually starting in young adults, causing a life-long burden for the patients. CD may occur anywhere in the gut from the mouth to the anus. Currently $80 \%$ of the patients must undergo at least one (often disabling) surgical removal of an intestinal segment [1]. Given the extent of morbidity and the unsatisfactory treatments available, research on pathogenesis as well as factors triggering disease flares is intense. In addition, CD has become a "prototype disease" for chronic autoinflammatory disorders with a polygenic background and multi-facetted, environmental trigger components. Environmental factors contribute to both pathogenesis and disease flares. Therefore, $\mathrm{CD}$ is a disease par excellence to study the interactions between host genetics, environmental factors, and "in-vironmental" factors-for example, our intestinal microbiome which is under control of our innate immune system.

We have entered a period of 'systems medicine' where the power of highly parallel sequencing and other 'omic technologies can be used to interrogate the mechanisms of human disease. CD led the field of polygenic disease genetic susceptibility discovery through the identification of mutations of Nucleotide-binding Oligomerisation Domain protein 2 (NOD2; CARD15) in 2001 that confer susceptibility to CD [2]. Judy Cho's group that now presents another interesting manuscript in "Genes and Immunity" was the first (together with another group) to demonstrate the impact of NOD2 variants [3, 4]. Interestingly, NOD2 is an innate immune receptor, a receptor for muramyl dipeptide-which is a component of the bacterial cell wall. NOD2 mutants associated with susceptibility to $\mathrm{CD}$ are

Gerhard Rogler

gerhard.rogler@usz.ch

1 Department of Gastroenterology and Hepatology, University of Zurich, 8091 Zurich, Switzerland deficient in their recognition of bacterial wall products. Pattern recognition receptors are highly expressed in intestinal macrophages and epithelial cells-pointing to the importance of microbiota recognition by cells of the intestinal innate immune system [5].

During the last 15 years, tremendous advances have been made in determining further genetic risk factors for IBD. So far, more than 170 genetic risk factors have been identified [6]. Many IBD loci are shared with other inflammatory diseases, such as ankylosing spondylitis, psoriasis, diabetes, or lupus [7].

Whilst alterations in the human genome coding relevant variant protein products have most likely not emerged significantly over the last 50 years, the incidence of $\mathrm{CD}$ has dramatically increased in Western countries [8] and more recently in the Asian-Pacific area [9]. Unfortunately, the determination of the presence of risk genes does not allow predicting disease courses or clinical phenotypes in $\mathrm{CD}$ patients. Genotyping is not sufficient to understand the pathogenesis of CD. Functional impact for innate and adaptive immunity has been studied for a number of specific genetic variants; however, the selection usually was arbitrary. Gettler and co-workers in this issue of "Genes and Immunity" have used an "unbiased approach". They used a gene prioritization regression model (GPRM) by integrating complementary mRNA expression datasets (RNA-Seq data only from the terminal ileum of 302 newly diagnosed, untreated $\mathrm{CD}$ patients and controls). Transcriptome-wide associations and co-expression network analyses were performed on those ileal RNA-Seq datasets. By this approach, Judy Cho's group identified 40 genome-wide significant genes. Further co-expression network analysis identified "a single gene module" that was highly enriched for CD-locus genes. This "gene module" was most highly expressed in monocytes/macrophages. The authors find "the marked enrichment of genes ... within monocytes compared to dendritic cells ...unexpected". In fact, we do not. Monocytes/macrophages have been shown to be of central importance in $\mathrm{CD}$ pathogenesis in the past. With respect to 
their transcriptional profile they differ from macrophages in other tissues, such as Kupffer cell in the liver, alveolar macrophages, or osteoclasts [10]. "Normal" intestinal macrophages show a specific phenotype with low expression of typical monocyte antigens, such as CD14 or CD16 [11]. They display an "anergic", "regulatory", "tolerogenic", or M2 phenotype with also low expression of costimulatory molecules and low expression of pattern recognition receptors such as TLR4 or TLR2 [12]. The functional characteristics of intestinal macrophages are altered in CD patients [12]. In actively inflamed CD mucosa the heterogeneity of the intestinal macrophage population is strongly increased. The new data provided by Gettler et al. perfectly fit into this picture. They will be very helpful to study the pathogenic role of the altered intestinal macrophage population in $\mathrm{CD}$ in more detail. However, they also need to be translated now in functional studies in this cell population to lead to new therapeutic approaches. This finally will allow to selectively target pathways that are specific for $\mathrm{CD}$ pathogenesis. In this light the presented manuscript is important and should stimulate researchers working on that cell population which (after the hype about dendritic cells) experiences a "renaissance".

\section{Compliance with ethical standards}

Conflict of interest GR was consultant to Abbvie, Augurix, Calypso, Cellgene, FALK, Ferring, Essex/MSD, Genentech, Janssen, Lilly, Novartis, Pfizer, Roche, UCB, Takeda, Tillots, Vifor, and Zeller. GR has received speaker's honoraria from Abbvie, FALK, Ferring, MSD, Pfizer, Phadia, Takeda, Tillots, UCB, and Vifor and received educational grants and research grants from Abbvie, Ardeypharm, FALK, Flamentera, Novartis, MSD, Pfizer, Roche, Takeda, Tillots, UCB and Zeller. MH declares that he has no conflict of interest.

Publisher's note: Springer Nature remains neutral with regard to jurisdictional claims in published maps and institutional affiliations.

\section{References}

1. Rieder F, Fiocchi C. Intestinal fibrosis in inflammatory bowel disease-current knowledge and future perspectives. J Crohns Colitis. 2008;2:279-90.

2. Hugot JP, Chamaillard M, Zouali H, Lesage S, Cezard JP, Belaiche J, et al. Association of NOD2 leucine-rich repeat variants with susceptibility to Crohn's disease. Nature. 2001;411:599-603.

3. Cho JH. The Nod2 gene in Crohn's disease: implications for future research into the genetics and immunology of Crohn's disease. Inflamm Bowel Dis. 2001;7:271-5.

4. Bonen DK, Ogura Y, Nicolae DL, Inohara N, Saab L, Tanabe T, et al. Crohn's disease-associated NOD2 variants share a signaling defect in response to lipopolysaccharide and peptidoglycan. Gastroenterology. 2003;124:140-6.

5. Rogler G. The effects of NOD2/CARD15 mutations on the function of the intestinal barrier. J Crohns Colitis. 2007;1:53-60.

6. Lee JC, Biasci D, Roberts R, Gearry RB, Mansfield JC, Ahmad T, et al. Genome-wide association study identifies distinct genetic contributions to prognosis and susceptibility in Crohn's disease. Nat Genet. 2017;49:262.

7. Richard-Miceli C, Criswell LA. Emerging patterns of genetic overlap across autoimmune disorders. Genome Med. 2012;4:6.

8. Molodecky NA, Soon IS, Rabi DM, Ghali WA, Ferris M, Chernoff $\mathrm{G}$, et al. Increasing incidence and prevalence of the inflammatory bowel diseases with time, based on systematic review. Gastroenterology. 2012;142:46-54 e42.

9. Ahuja V, Tandon RK. Inflammatory bowel disease in the AsiaPacific area: a comparison with developed countries and regional differences. J Dig Dis. 2010;11:134-47.

10. Gordon S, Pluddemann A. Tissue macrophages: heterogeneity and functions. BMC Biol. 2017;15:53.

11. Rogler G, Hausmann M, Vogl D, Aschenbrenner E, Andus T, Falk W, et al. Isolation and phenotypic characterization of colonic macrophages. Clin Exp Immunol. 1998;112:205-15.

12. Hausmann M, Kiessling S, Mestermann S, Webb G, Spottl T, Andus T, et al. Toll-like receptors 2 and 4 are up-regulated during intestinal inflammation. Gastroenterology. 2002;122:1987-2000. 